\title{
Non-Fickian diffusion in biomaterials
}

\author{
Almeida, Giana ${ }^{a^{*}}$; Perré, Patrick ${ }^{b}$ \\ a Ingénierie Procédés Aliments, AgroParisTech, INRA, Université Paris-Saclay, 91300, Massy, \\ France. \\ b LGPM, CentraleSupélec, Université Paris-Saclay, 8-10 rue Joliot-Curie, 91190 Gif-sur-Yvette, \\ France.
}

*E-mail of the corresponding author: giana.almeida@agroparistech.fr

\begin{abstract}
The knowledge of water vapour diffusion in biomaterials is very important in several fields of application (drying, building materials, food packaging...). The process of vapour water transfer in biomaterials is usually described by Fick's law of diffusion. Nevertheless, sometimes the experimental data of transient experiments in biosourced materials do not respect the standard Fickian model.

The precise determination and formulation of this non-Fickian behaviour is therefore an important topic. This work presents a new methodology to quantify the non-Fickian diffusion. Based on memory functions and imbedded in a comprehensive heat and mass transfer computational model, the method is robust and prone to be applied to different biomaterials. Examples of application are proposed in the case of biofilms and lignocellulosic materials.
\end{abstract}

Keywords: Biofilm; Low Density Fiberboard; Modeling; Non-Fickian diffusion; Wood 


\section{Introduction}

A detailed understanding of water vapour transfer in biomaterials is very important in several fields of application (drying, building materials, food packaging...). Regarding the drying process, the value of mass diffusivity, as well as its variations with moisture content, governs the second drying period, which is the most important in terms of drying time, energy consumption and product quality. As they are exposed to cyclic changes in relative humidty of the air, inducing cyclic changes in moisture content and bound water transfer, biomaterials used in building are always in transient regime. Finally, in the case of biofilms used as barrier in food packaging, the transfer properties are among the most important physical properties to ensure a good end-use.

Nevertheless, experimental data of water vapour uptake of some biomaterials showed that did not follow the standard Fickian model. In these works, a phenomenon of slowing down the process of mass transfer at the final stage, often called non-Fickian behaviour was observed [1,2]. In polymer science, this phenomenon is attributed to molecular relaxation, either at a local scale or at a global scale, in relation with stress relaxation $[3,4,5]$. The precise determination of this non-Fickian behaviour showed to be a difficult task since the physical phenomena causing this non-Fickian behaviour is different depending on the biomaterial structure and physical properties.

This work presents a new methodology to quantify the non-Fickian diffusion very robust and prone to be applied to different biomaterials. Examples of application of this methodology on porous [6] and amorphous polymer [7] will be presented. The final part of this paper is devoted to abnormal behaviours, due to dual-scale effects, observed at short times in the case of low density fiberbroards (LDF) [8].

\section{Materials and Methods}

\subsection{Materials}

Vapour sorption experiments were applied on two types of biomaterials: porous material (wood, thermal-treated wood, Low density Fiberboard) and amorphous polymer (biofilms).

Soprtion tests have been performed on native and thermally modified European beech (Fagus sylvatica L.). Thermally modified wood samples were submitted to a temperature level of $220^{\circ} \mathrm{C}$ during $60 \mathrm{~min}$ [6]. The oven dry density of unmodified and thermally modified wood was 680 and $655 \mathrm{~kg} / \mathrm{m}^{3}$, respectively. The interested reader can refer to the reference [6] for a detailed description of the experiments. 
Concerning biofilms, neat PLA (Polylactide) films were prepared using the casting method described in [9]. Biofilms were $60-80 \mu \mathrm{m}$ thick, with a standard deviation of $20 \%$. They were stored over $\mathrm{P}_{2} \mathrm{O}_{5}$ at room temperature prior to sorption tests.

Prior to the sorption tests, the LDF samples was stabilised to an equilibrium moisture content close to $12 \%$. The disk-shaped samples, with a diameter of $72 \mathrm{~mm}$, were cut from the equilibrated material from $20-\mathrm{mm}$ thick panels. An epoxy resin was applied to the lateral face of the sample to block the surface porosity. Because of the propensity of fibreboard samples to absorb the epoxy resin, the resin was first applied to a thin strip of aluminium, which was then applied around the sample.

\subsection{Sorption tests}

\subsubsection{Sorption tests applied on wood samples}

The samples were firstly stored in containers with controlled air temperature of $20^{\circ} \mathrm{C}$ and relative humidity of ca. 35\% during 6 months, wich allowed obtaining their uniform initial bound water content. Then the samples were transferred to the set-up with controlled air parameters, i.e. temperature of $23.5^{\circ} \mathrm{C}$, relative humidity of $75.5 \%$ and the forced air flow of $0.8 \mathrm{~m} / \mathrm{s}$. The final bound water content of each sample was determined by the gravimetric method after the sorption experiment. More details about the vapour mass sorption results can be found in [6].

\subsubsection{Sorption tests applied on biofilms}

Water vapour sorption was performed by using dynamic vapour sorption with a DVS intrinsic apparatus (Surface Measurement Systems, London, UK). A support pan was specially designed to allow the entire film surface to be available for the vapour exchange. Approximately $20 \mathrm{mg}$ of sample was placed in the sample pan. The samples were first equilibrated at $0 \% \mathrm{RH}$ over $360 \mathrm{~min}$. The amount of dry matter was determined at the end of this plateau. Then, the RH was increased to $30 \%$ and maintained at this level for 1440 min. Finally, the RH was increased to $90 \%$ and maintained at this level for $2880 \mathrm{~min}$. Preliminary tests confirmed that the duration of each RH plateau was suficient to attain the equilibrium condition (the rate of change of mass per unit time $(\mathrm{dm} / \mathrm{dt})$ was less than $0.0005 \% / \mathrm{min}$ ). The data were saved every $20 \mathrm{~s}$. More details about the methodology are available in [9].

\subsubsection{Sorption tests applied on $L D F$}

LDF samples were tested according to a new method proposd in [8]. The principle of this method is to impose a sudden change in $\mathrm{RH}$ on one side of the sample to create a transient 
diffusive flux through the sample. The RH measured on the back face of the sample provides the information from which the mass diffusion coefficient is determined by inverse analysis. To that purpose, a specific device was conceived and built to obtain quasi 1-D transfers along the sample thickness. This requires the back face chamber, where the $\mathrm{RH}$ is measured, to be perfectly airtight and as small as possible [8].

\section{Mathematical model to determine the Non-Fickian diffusion}

Several effects taking place at the local, microscopic scale, have important effects at the macroscopic scale. In this section, we will treat two major effects encountered in bio-based building materials :

- molecular relaxation : due to the mobility of macromolecules, new sorption sites appear due to the reorganization of molecules after change of bound water content. For lignocellulosic materials, this is particularly observable at high levels of relative humidity or for thermally modified products $[8,10]$,

- dual scale effects : due to the long characteristic time of diffusion in the secondary cell wall, the local equilibrium sometimes fails. At the macroscopic level, this induces a delay between the change of air characteristics and the moisture content of the product, even at the very fine microscopic scale.

\subsection{Molecular relaxation}

This phenomenon can be approached using a relaxation function, defined by a sudden change of RH surrounding the product at the microscopic scale, from $R H_{i n \boldsymbol{i}}$ to $R \boldsymbol{H}_{\text {in }}$, assuming the cell wall to be at equilibrium at $t=0\left(X_{i n i}=X_{e q}\left(R H_{i m i}\right)\right)$. In this case, the evolution of the local moisture content is simply the signature of molecular relaxation:

$$
X(t)-X_{i n i}=\Delta X_{e q} \times \varphi(t)
$$

where $\Delta X_{e q}$ is defined by the sorption isotherm values $\Delta X_{e q}=X_{e q q}\left(R H_{f i n}\right)-X_{e q}\left(R H_{m i}\right)$ and $\varphi$ a monotonic function which respects $\lim _{\varphi}(t)_{t} \infty x_{i}=1$.

In a more general case, the value of $\boldsymbol{R H}$ varies continuously in time, so that a convolution product is required :

$$
X(t)-X_{i n i}=\int_{0}^{t} \varphi(t-\tau) \frac{\partial X_{e q}}{\partial \tau} d \tau
$$


It is obvious from equation (1) that if $\varphi$ is the casual function (the Heaviside function, see Fig. 1), any memory effect vanishes.

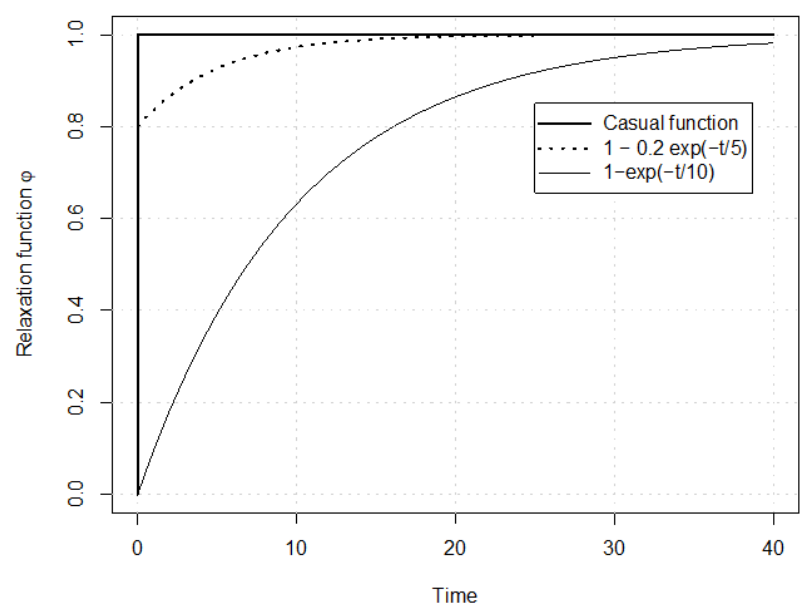

Fig. 1. Some possible shapes of the relaxation function.

\subsection{Dual scale effects}

Similar effects can arise from dual scale phenomena : depending on the characteristic time for the moisture field to equilibrate at the microscopic scale, a delay might exist between the equilibrium moisture content and the actual moisture content. This is know as the failure of the local thermodynamic equilibrium. For the sake of example, we can refer to mass diffusion in a cylindrical fiber of radius $a$. By neglecting the resistance to mass transfer in the gaseous phase of the porous medium, the equilibrium moisture content $X_{\mathbf{e q}}$ can be used as Dirichlet boundary conditions at the solid phase surface. In the case of a perfect cylindrical shape for example, the corresponding analytical solution reads [11]:

$$
X(r, t)-X_{i n i}=\Delta X_{e q} \times\left(1-\frac{2}{a} \sum_{n=1}^{\infty} \frac{\exp \left(-D \alpha_{n}^{2} t\right) J_{0}\left(r \alpha_{n}\right)}{\alpha_{n} J_{1}\left(a \alpha_{n}\right)}\right)
$$

Where the values of $\alpha_{n}$ are the positive roots of

$$
J_{0}\left(a \alpha_{n}\right)=0
$$


Where $J_{0}(x)$ and $J_{1}$ are the Bessel functions of the first kind of order zero and one. The integration of equation (3) over the cylindre volume allows the average moisture content $\bar{X}$ to be obtained :

$$
\bar{X}(t)-X_{i n i}=\Delta X_{e q t} \times\left(1-\sum_{n=1}^{\infty} \frac{4}{a^{2} \alpha_{n}^{2}} \exp \left(-D \alpha_{n}^{2} t\right)\right)
$$

This equation is very similar to the equation obtained in the case of molecular relaxation (2). A function $\varphi$ can be therefore be deduced from equation (5):

$$
\varphi(t)=1-\sum_{n-1}^{\infty} \frac{4}{a^{2} \alpha_{n}^{2}} \exp \left(-D \alpha_{n}^{2} t\right)
$$

\subsection{Computational solution}

The previous sections tells us that equation (2) is a general expression allowing the nonFickian behaviour to be accounted for in the macroscopic formulation. The kernel function $\varphi$ is therefore the signature of the product behaviour and its history can be expressed as the form of a convolution product that account for the time evolution of the conditions undergone by the product. This formulation was embedded in the coupled heat and mass transfer code TransPore, a custom computational model currently used in our team as comprehensive physical engine to perform inverse analysis $[8,12]$.

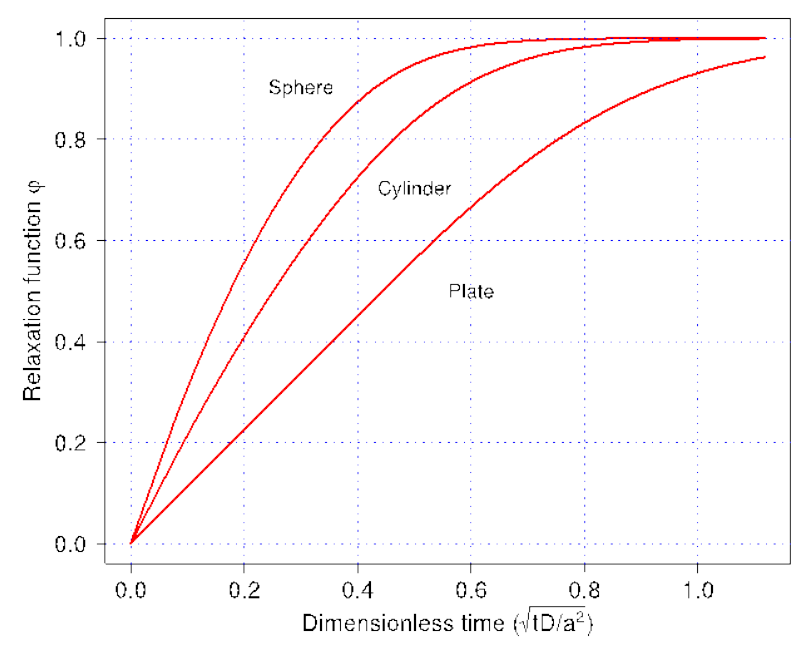

Fig. 2. Dimensionless change of moisture content. Result obtained for different shapes using the first 500 terms of the respective analytical solutions [11]. 


\section{Results}

The water vapour uptake of the biomaterials studied can be observed in Figs 3 and 4. Each figure contains experimental results and predicted bound water content changes as a function of the square root of time simulated by the formulation presented in the previous section.

Fig. 3 gives a clear vision of transient water vapour transfer in native wood (left) and thermally treated wood (right). The parameter identification allows the sorption behaviour to be represented as a combination of Fickian and non-Fickian behaviour. The red line stands for the full model whereas the blue line was obtained by cancelling the relaxation function (its value was set constant at $\varphi(t)=\varphi(0), \forall t$ ). The effect of thermal treatment on the wood/water relations is obvious in the plots : significant reduction of the sorption isotherm [13] and considerable increase of the non-Fickian part. Olek et al. [6] attributed this non-Fickian behaviour to the chemical changes in wood components (hemicellulose, cellulose and lignine) caused by thermal treatment.
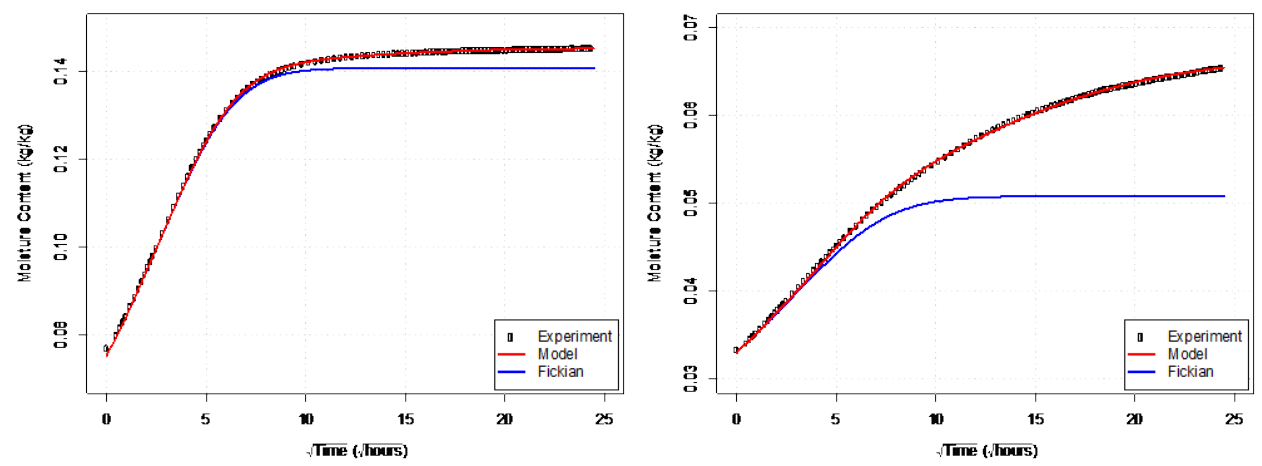

Fig. 3. Sorption tests and theoretical analysis for native (left) and thermally treated beech wood (right). Note the impressive increase of the non-Fickian behavior in treated wood (after Olek et al. [6])

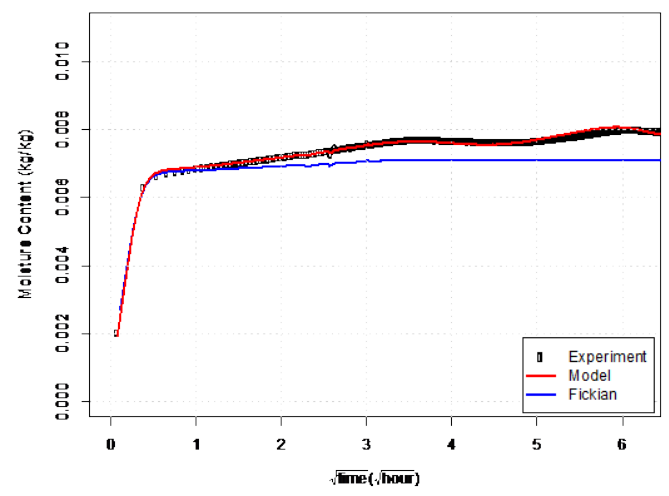

Fig. 4. Sorption tests and theoretical analysis for PLA. (after Almeida et al. [7]). 
Table 1 list the diffusion parameters identified by the model: Diffusion coefficient, following the Fickian diffusion law $\left(\mathrm{D}_{0}\right)$, total amount of bound water molecules allowed by relaxation and reorganization of already sorbed water at infinite time $(\varphi(0))$ and time constant of relaxation $(\boldsymbol{\tau})$. According to these parameters, thermal modification alters the diffusion behavior of wood in two characteristics: (a) significant reduction of diffusivity, (b) dramatic increase of the non-Fickian part [6]. For PLA, the determined diffusion coefficient $\left(\mathrm{D}_{0}=5.7 \times 10^{-12} \mathrm{~m}^{2} \cdot \mathrm{s}^{-1}\right)$ is in the range of published data $[5,14]$. Works in progress depicts also a large increase of the non-Fickian proportion when the PLA film is charged with nanostructures.

Table 1. Fickian and non-Fickian Diffusion parameters determined by the model

\begin{tabular}{lccc}
\hline \multicolumn{1}{c}{ Biomaterial } & $\mathbf{D}_{\mathbf{0}}\left(\mathbf{m}^{\mathbf{2}} \mathbf{s}^{-1}\right)$ & $\varphi(0)$ & $\boldsymbol{\tau}(\mathbf{s})$ \\
\hline Unmodified wood $^{\mathrm{a}}$ & $0.58 .10^{-10}$ & 0.065 & $5.35 .10^{5}$ \\
Thermally treated wood $^{\mathrm{a}}$ & $0.38 .10^{-10}$ & 0.47 & $7.65 .10^{5}$ \\
PLA $^{\mathrm{b}}$ & $0.057 .10^{-10}$ & 0.18 & $0.67 .10^{5}$ \\
data from Olek et al. $[6] ;{ }^{\mathrm{b}}$ Almeida et al. $[7]$.
\end{tabular}

The final part of this paper is devoted to transient diffusion in LDF (160 kg.m $\left.{ }^{-3}\right)$. This kind of materials, used for insulation in construction, presents an open porosity that allows a rapid diffusion at the macroscopic level. Therefore, in spite of the huge contrast between the macroscopic dimension (thickness in buildings) and the microscopic solid phase (the order of one or some natural fibers) the characteristic time diffusion of the micro- and macro-scale overlaps. This induces a non-local equilibrium and alter the macroscopic behavior. In this case of open porosity, the macroscopic mass transfer is expressed as follows :

$$
q_{v}=-\rho_{g} f D_{v} \nabla \omega_{v}
$$

Where $D_{\boldsymbol{v}}$ is the diffusivity of water vapour in air and $f$, the product parameter, represents the dimensionless coefficient of resistance $(0<f<1)$. In order to focus on the dual-scale effect and to avoid the relaxation effect, we focus here on the first hours of diffusion (Fig. 5). When using the usual macroscopic formulation, yet accounting rigorously for the coupling between heat and mass transfer, an unrealistic value of $f(2.5)$ is needed for the model to fit the experimental data. This was already discussed in [8]. Using a relevant value of $f=0.5$, as predicted by homogenisation from the real 3D morphology [15], the model predict a too low dynamic of RH increase at the back face. Finally, the use of a simple 
$\varphi$ function, whose parameters were determined by identification, the dynamic of $\mathrm{RH}$ evolution can be captured with the same value of $f(0.5)$. Additional works are in progress in our team to further test this new approach. It seems in particular that this new formulation allows both the $\mathrm{MC}$ and the $\mathrm{RH}$ at the back face to be simulated with the same set of parameters.

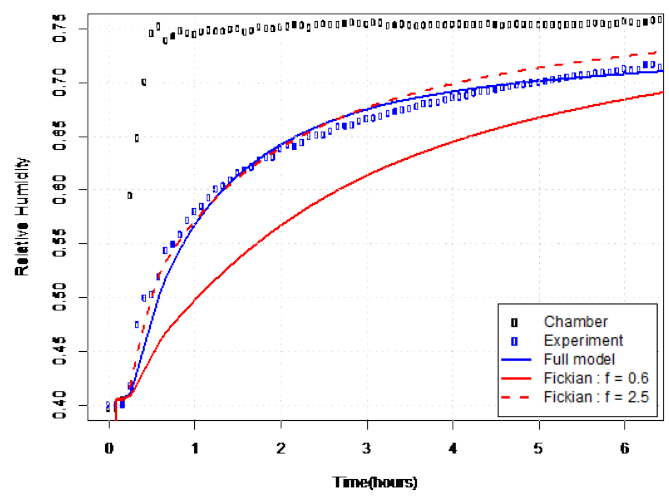

Fig. 5. The back-face method with LDF: experimental RH value at the back face of the sample and computed evolution obtained with various assumptions (data after Perré et al. [8]).

\section{Conclusion}

This paper presents a geral approach to account, at the macroscopic level, for various situations of non-Fickian diffusion in biosourced products. This model is applied to experimental data of water vapour uptake of biomaterials presenting completely different physic-chemical structure (porous biomaterials with different morphologies and biopolymer films). The proposed model is able to describe the diffusion behavior of such biomaterials and differences obtained are discussed. It is proved that the non-Fickian diffusion must be taking into account for such materials in order to insure its proper transformation and good end-use.

\section{References}

[1] Wadsö L. Describing non-Fickian water-vapour sorption in wood. J Mater Sci 1994, 29(9), 2367-72

[2] Englund, E.T.; Thygesen L.G.; Svensson, S.; Hill C.A.S. A critical discussion of the physics of wood water interactions. Wood Sci Technol 2013, 47(1), 141-61

[3] Crank, J.A. theoretical investigation of the influence of molecular relaxation and internal stress on diffusion in polymers. J Polym Sci 1953;11(2):151-68

[4] Christensen, GN. Sorption and swelling within wood cell walls. Nature, 1967, 213(5078):782-4 
[5] Davis, E. M.; Minelli, M.; Baschetti, M.G.; Elabd, Y.A. Non-Fickian Diffusion of Water in Polylactide. Industrial \& Engineering Chemistry Research, 2013, 52(26), 8664-8673

[6] Olek, W.; Rémond, R.; Weres, J.; Perré, P. Non-Fickian moisture diffusion in thermally modified beech wood analyzed by the inverse method. International Journal of Thermal Sciences, 2016, 109, 291-298

[7] Almeida, G.; Domenek, S.; Perré, P. A New Mathematical Model to Quantify the Non-Fickian Diffusion on biofilms. under preparation.

[8] Perré P., Pierre F., Casalinho J., Ayouz M., 2015 - Determination of the mass diffusion coefficient based on the relative humidity measured at the back face of the sample during unsteady regimes, Drying Technology, 33: 1068-1075.

[9] Espino-Pérez, E.; Bras, J.; Almeida, G.; Plessis, C; Belgacem, N.; Perré, P; Domenek, $\mathrm{S}$. Designed cellulose nanocrystal surface properties for improving barrier properties in polylactide nanocomposit. Carbohydrate Polymers, 2018, 183, 267-277.

[10] Olek, W.; Perré, P.; Weres, J. Implementation of a relaxation equilibrium term in the convective boundary condition for a better representation of the transient bound water diffusion in wood, Wood Sci. Technol., 2011, 45: 677-691.

[11] Crank, J. The mathematics of diffusion. Oxford University Press, 1975.

[12] Perré, P.; Turner, I. A 3-D version of TransPore : a comprehensive heat and mass transfer computational model for simulating the drying of porous media, Int. J. Heat Mass Transfer, 1999, 42: 4501-4521.

[13] Almeida, G.; Brito, J.; Perré, P. Effect of thermal treatment on wood/water relationships, as defined on minute samples, Holzforchung, 2009, 63: 80-88.

[14] Martinez-Sanz, M.; Lopez-Rubio, A.; Lagaron, J. M. Optimization of the Dispersion of Unmodified Bacterial Cellulose Nanowhiskers into Polylactide via Melt Compounding to Significantly Enhance Barrier and Mechanical Properties. Biomacromolecules, 2012, 13(11), 3887-3899

[15] Louërat, M.; Ayouz, M.; Perré, P., Heat and moisture diffusion in spruce and wood panels computed from 3-D morphologies using the Lattice Boltzmann method, Int. J. Thermal Science, under revision. 